\title{
SOME CONSIDERATIONS ON THE PHYSIOLOGY OF THE OTIC LABYRINTH*
}

\author{
J. GORDON WILSON, M.D., AND F. H. PIKE, M.D. \\ CHICAGO \\ NEW YORK
}

The philosopher's statement, now more than a century old, that there is nothing in the mind that has not come in through the senses, might be applied to motion in general. Certainly, so far as those movements due to the central nervous system are concerned, we may say that there are extremely few motions that have not had their antecedent afferent impulses. The modern conception of the nervous system regards it as a mechanism in which afferent impulses, arising in the various kinds of peripheral receptors or sensory endings are gathered up into a definite body of sensory impulses, and somewhere in their course, are summed or integrated to a definite orderly motor response.

For example, when a child is learning to write, and is copying a simple figure, afferent impulses are passing in from various peripheral sources, not from the eye alone, but also from the skin, and the muscles which feel and grasp the pencil, and from other sources. The actual movements of the pencil in the fingers are in response to all these impulses, some of which make their way to the cerebrum directly, and others, particularly those which deal with the "unconscious sensation" of the muscles, pursue an indirect path through the cerebellum. But all these afferent impulses, from whatever source or over whatever path, must be associated or added together and harmonized - integrated - within the brain before the fingers can be moved adequately.

The labyrinth of the ear is a circumscribed, definitely located peripheral sense organ, and one task of modern physiology is to inquire into (1) the modes of stimulation of the particular forms of sensory endings which are found in the labyrinth, (2) the effect of the normal afferent impulses from the labyrinth on the normal motor response of the animal, and (3) the particular variations in this motor response occasioned by afferent impulses of unusual violence, e. $g$.. those arising from artificial stimulation or from pathological processes within the ear, or by total lack of such afferent impulses following experimental or pathological loss of the labyrinth.

* Submitted for publication Sept. 29, 1914.

* Based on a paper read before the Section on Neurology of the New York Academy of Medicine, Feb. 11, 1913. 
Obviously, the detailed consideration of all these matters would transcend the limits of such a paper as the one we now purpose to present, and we will consider, therefore, certain salient points which have impressed themselves on us in the course of a long and rather detailed study of the subject. ${ }^{1}$

Exact experimental work on the semicircular canals began with Flourens in 1828, although other investigators, notably Purkinje, had stimulated the canals in various ways without knowing exactly with what structures they were dealing. Flourens, while experimenting on pigeons, noticed that after removal of one canal, movements of the head occurred in the plane of the destroyed canal, or, as it is sometimes expressed, about an axis perpendicular to the plane of the destroyed canal. Flourens' work has been repeated by many subsequent observers, and the results may now be regarded as firmly established. These specific effects of experimental lesions of the semicircular canals are not due, as some have supposed, to accidental injury to the cerebellum, or to the extension of inflammatory processes to it. Nor does experimental removal of the cerebellum, without injury to the labyrinth or to the vestibular nerves, in our experience on mammals result in exactly the same motor disturbances as elimination of the labyrinth.

In pigeons, removal of one labyrinth is followed in ten to fourteen days by torsion of the head. But removal of the cerebellum in part or wholly, is not followed by such torsion, but by tremors. Removal of the cerehellum and then removal of the labyrinth is followed immediately by the onset of torsion, and the less the amount of cerebellar tissue removed, the greater the delay in the onset of the torsion. This indicates that in pigeons, so far as torsion is concerned, there is something in the cerebellum which resists the onset of torsion, and removal of the cerebellum does not impede its onset. In other words, torsion is essentially an extracerebellar phenomenon. This is quite in keeping with our observations on mammals, with reference to torsion and nystagmus as well. ${ }^{1}$

Removal of the cerebellum in the pigeon, without injury to the labyrinth is, as Lange points out, a matter of some difficulty. Obviously, unless we can get lesions, restricted to the labyrinth without injury to the cerebellum on the one hand, and lesion of the cerebellum alone without involvement of the labyrinth or vestibular tract on the other, we can get no clear or exact picture of the effects of such lesions, and hence no exact basis for a differential diagnosis of pathological lesions of these two mechanisms.

1. Wilson and Pike: Philosophical Trans. Royal Soc., London, Series B, 1912, cciii, 127.

2. Lange: Arch. f. d. ges. Physiol, 1891, 1, 615. 
Experimental elimination of the labyrinth in the pigeon and many other animals has been accomplished (1) by anatomical removal of the labyrinth or division of the vestibular nerve, and (2) by anesthetizing the labyrinth with cocain. Stimulation of the labyrinth has been done by electrical currents, temperature, ice, hot and cold water, hot wire, etc., mechanical pressure, withdrawal of water by crystallin substances applied to the membranous labyrinth, and rotation of the body and particularly of the head, about an axis perpendicular to the plane of some one set of canals. The various forms of stimuli may be classified according to their effects as strong or weak. The stronger stimuli, e. g., closure of the electrical current when the cathode or negative pole is over the labyrinth, hot water, in certain positions of the head in space, strong pressure on the endings of the vestibular nerve, or a fluid current set up either by mechanical pressure on the canal or by placing a crystal of cocain on the horizontal canal in such a position that the current is toward the ampulla, cause a movement of the head and eyes in the plane of the canal and arvay from the stimulated side. More moderate stimuli, e. g., the closure of the electrical current when the anode is over the labyrinth, cold water or ice, again with due reference to the position of the head, and withdrawal of water in such a way that the current is away from the ampulla (horizontal canal), cause movement of the head and eyes in the plane of the stimulated canal toward the stimulated side.

The extremely slow currents set up by the slow withdrawal of water from the canals must not be confused with the strongly flowing currents sometimes supposed to be set up in the semicircular canals by change of aspect of the head in space or by irrigation of the external auditory meatus with hot and cold water.

Much discussion has arisen as to whether the effects of unilateral anatomical removal are due to simple absence of one labyrinth, or to irritation of the nerve endings and nerve trunk in the operation wounds. We may safely say that irritation plays but little part in the effects of anatomical removal since (1) the same results are obtained when the labyrinth is anesthetized with cocain, and (2) the results of irritation, if any be present, are the same as those observed after application of the weaker stimuli to the labyrinth, and (3) the results of removal of the second labyrinth after an interval are much less severe than those observed after removal of the first labyrinth. The results are, in the main, due to the unbalanced action of the intact labyrinth.

Without at this time going into a detailed review of the evidence on which the statement rests, we may say that the effects of elimination of the left labyritth, either by anatomical removal or anesthesia by 
cocain, are the same as those following electrical stimulation of the labyrinth with the anode over the mastoid fossa, by the application of cold water to the ear, or by rotation to the right. We may, then, consider at this time, the specific results of experimental lesions of the labyrinth, of anesthesia, of electrical and thermal stimulation and of rotation, and afterward consider certain theories of the action of the labyrinth.

In point of historical development, the first most important work goes back to Purkinje whose laws of rotation vertigo, as they are now called, were worked out before Flourens did his experimental removals. Although Purkinje's observations were correct in every essential particular, he did not attribute the results to the semicircular canals, but supposed that in rotation the brain exerted traction on certain cranial nerves because it lagged behind the skull in starting or stopping. We may give these laws of rotation vertigo in detail.

We should distinguish between the period during which rotation actually occurs-the rotation period proper-and the period beginning immediately after the cessation of rotation-known as the postrotatory period. If one stands erect, or sits erect in a chair, and rotates, say to the right, objects may apparently move to the left and one has a sensation of real movement to the right. Shutting the eyes excludes the apparent movement of external objects, but does not affect the sensation of movement of one's own body. If one stands erect and rotates about a vertical axis for some minutes and then stops, surrounding objects still appear to move around and vertigo may be present in the postrotatory period. The direction of the apparent motion of oneself depends on the directions of the preceding real motion and is always opposite in direction. If one bends the head forward 90 degrees and then rotates, the apparent movement during the postrotatory period is about a vertical axis. But if, after again bending the head forward and rotating, the head is raised to an erect position during the post rotatory period, the apparent movement is no longer about a vertical but about a horizontal axis. Objects will seem to rise on one side and fall on the other. The axis about which apparent movement occurs is always the axis of the head about which the preceding real rotation occurred. Hitzig, in his classical paper, gave the first clear and accurate account of the results of electrical stimulation of the labyrinth-galvanic vertigo - and showed that these results fall into line with those of rotation vertigo. When the cathode is placed over the left fossa mastoidea and the anode over the muscles of the neck, the eyes move slowly to the right and quickly back to the left. The subject may get the impression of apparent movement of his own body to the left; the apparent movement of external objects is frequently to the left, but varies with the 
individual. Closing the eyes abolishes the impression of apparent movement of external objects, but does not abolish the sensation of apparent movement of one's own body. As Hitzig expressed the relation between stimulating electrode and direction of eye movement, the quick movement of the eyes is in the direction of flow of the electrical current, i. e., in the case just cited, toward the cathode.

Hitzig $^{3}$ was among the first to stimulate the labyrinth with cold, although he did not know at the time that it was the labyrinth he was stimulating. Bornhardt, ${ }^{4}$ a few years later, employed the direct application of heat and cold to the labyrinth as stimulating agents. Goltz ${ }^{5}$ referred to the disturbance of equilibrium and the eye movements following incautious irrigation of the external auditory meatus, especially when the tympanic membrane was ruptured, as being common knowledge in his day, and attributed the effects to stimulation of the semicircular canals and the labyrinth generally by the water. From these early beginnings, there has grown up the caloric test as applied in clinical practice of the present day, largely due to the work of Bárány.

Anatomical removal of the labyrinth in mammals, e. g., dogs and monkeys, is accompanied by certain characteristic phenomena. Certain of these are acute, lasting but a few days, while others persist as long as the animal lives. If one labyrinth only is destroyed, there is a slow movement of the eyes toward the injured side, and a quick return jerk to the median position. This nystagmus persists but a few days, and then wholly ceases, the eyes being then normal in position and capability for movement. In lower forms, such as the dog-fish ${ }^{6}$ and turtle, the eyes are permanently deviated toward the injured side, and there is no nystagmus.

Although the eyes in mammals appear to come back to the normal position in a few days, we have repeatedly observed that if the animal be placed in an abnormal posture, e. g., on its back, the symmetrical position of the eyes is altered and there will be observed a marked ventral deviation of the eye on the side of the lesion. This persists for weeks. The animal's head is permanently turned toward the injured side, and, in the early days of the postoperative period, the animal falls or rolls to the injured side. I.ater the animal becomes able to walk without falling, but turns toward the injured side so that it goes in a circle. In a few days more, it is able to walk a straight line, even when

3. Hitzig: Arch. f. Anat. u. Physiol., 1871, p. 771.

4. Bornhardt: Arch. f. d. ges. Physiol., 1876, xii, 471.

5. Goltz: Arch. f. d. ges. Physiol., 1870, iii, 192.

6. Jour. Physiol., 1894, xv, 311; 1895, xvii, 192.

7. Wilson and Pike: Proc. Soc. Exper. Biol. and Med., 1913, xi, 52. 
its eyes are covered. The transitory falling over of the animal to the side of the lesion and the persistent deviation of the head is, we believe, due to the over activity of the intact labyrinth, whereby the muscles, chiefly the extensors on the intact side, are acting more effectively.

If both labyrinths are removed at one time, there is nystagmus as before, but it is irregular in character and does not persist as long as after unilateral removal. There is no torsion of the head to one side or the other, or at most, a comparatively slight inclination. The animal refuses to stand, but lies with head and feet outstretched and paws clutching the floor. If the head is raised voluntarily, there are coarse swaying movements reminding one of the swaying of a reptilian head. Food is not accurately grasped with the jaws. In attempting to take food, the nose is thrust to one side, above or below the desired object, only rarely touching the object squarely. A turtle deprived of both labyrinths may die of starvation because of its inability to seize food. In the dog and cat, the swayirg movements cease in two weeks or less. and the accuracy of grasp returns. Gradually, also, the cat or dog learns to walk, falling to one side or the other in the first attempts, and turning now to one side, now to the other in its efforts to cross a room. Finally, all these symptoms disappear, and it would be difficult for one to detect any motor disturbance in a dog with eyes unbandaged in walking across grass or a level floor, or in taking food.

The eye movements are of particular interest in connection with stimulation or removal of the labyrinth, and we may inquire into them in detail.

In all animals so far experimented on there is a slow movement of the eyes toward the injured labyrinth or toward the labyrinth to which the weaker stimulus is applied. In rotation, the eyes lag behind, going slowly to the side opposite to that toward which the body is rotated. In the postrotatory period the slow movement of the eyes changes and acquires the same direction as the preceding real rotation. The typical oscillatory movement of the eyes, alternately slow in one direction and quick in the opposite direction, is seen best in mammals. So far as our observation or knowledge goes, this is a characteristic type of nystagmus not seen in experimental lesions of the cerebellum or of any other portion of the central nervous system so long as the vestibular tract is intact. The slow deviation alone is seen in certain of the lower forms. We have found that the slow deviation alone is seen in decerebrated or partially anesthetized animals. Nystagmus in its characteristic form occurs only in those animals which have recovered to a considerable extent from the anesthetic, or in which the optic thalamus with the corpora striata and a considerable portion of the cerebral hemispheres remained. Some part of the mechanism which produces nystagmus 
must, therefore, be anterior to the corpora quadrigemina. The exact paths concerned will be reported on later.

Bartels, basing his views partly on the afferent nerve endings recently described in the eye muscles by Sherrington and his pupils, and partly on rotation experiments on infants, concludes that the quick return jerk of the eyes in labyrinthine nystagmus is a reflex dependent on some mechanism, probably cortical, but certainly cerebral. Bartels is not inclined to accept Bárány's view of a hypothetical Blickcentrum as the cause of nystagmus. Bartels found that in the first seven days of post partum life, rotation nystagmus was not obtainable. Later, it is obtainable when the' child is awake, but not during sleep. Prematurely-born infants do not exhibit rotation nystagmus for some time after birth. An infant born at the end of seven months, uterine life does not show rotation nystagmus in the early postpartum period, although anatomically, the semicircular canals are fairly well developed at the end of three months. The time of appearance of rotation nystagmus apparently depends more on the period elapsing after birth than on the absolute length of gestation.

We agree in the main with Bartels' idea of the mechanism of labyrinthine nystagmus, and have published additional evidence of their essential correctness and extended them considerably.

Clinicians and laboratory workers alike have been impressed with the quick phase of nystagmus, and the direction of nystagmus is usually given as the direction of the quick movement. In so doing, all have fixed attention on the mechanism which brings about the return of the eye to the normal position, and not on the lesion which causes the eye to deviate from its normal position. But the cause of the deviation from the normal position is the very thing which the clinician is most anxious to discover. If the experimenter has anything definite to say to the clinician, it is this one thing, that the cause of the slow deviation in labyrinthine nystagmus lies in the labyrinth or in the vestibular tract, and that the slow component of nystagmus is the one which it is most important to observe. The cause of the ocular deviation from the normal position may lie in other parts of the central nervous system than the labyrinth or the vestibular tract, and each may bring about the deviation in a particular way. The manner of return to normal is more likely to be uniform. Involvement of the labyrinth or the vestibular tract always, in experimental procedures at least, produces a relatively slow, wide deviation, in which both eyes move together, and we may repeat that labyrinthine nystagmus is characterized by these alternately slow and rapid movements. As at present used, nystagmus is an indefinite term, covering various types of ocular movements arising from lesions in diverse parts of the nervous system, 
and a more careful distinction of the different types of eye movement is greatly to be desired.

After the subsidence of the symptoms of the acute stage, certain other permanent defects are observable.

If but one labyrinth has been removed, there is a permanent torsion of the head to the injured side, and there may be a leaning, particularly of the anterior part of the body, to this side. This torsion of the head is much more marked in animals than in the human subject. In the latter, the torsion may be negligible. If both labyrinths have been removed, either at one sitting or at intervals, there is no marked torsion of the head or inclination of the body to one side. With eyes bandaged, the animal is unable to walk in a straight line, but goes now to one side, now to the other, or turns helplessly in a small circle. Ascending stairs offers no particular difficulty, but descending stairs usually results in a headlong fall. Jumping down from even a moderate height results in falling in a heap on the floor. An animal with one labyrinth intact walks in a straight line when blindfolded, ascends or descends stairs and jumps up or down without difficulty. In neither animal is there any loss of the sense of position of the muscles, so far as can be made out. The drop reflex - the thrusting out of the hind feet and legs when the animal is held vertically, head up, and rather rapidly lowered with the hands - is greatly depressed or wholly absent on the side of labyrinthine injury. In no case can any confusion concerning the relation of the parts of the body to each other, or to the body as a whole, be made out. The animal's mistakes and failures concern his relation to external objects or his orientation in space.

Such briefly and in general are the results of labyrinthine removal. Various theories have been put forward to explain these defects. We may mention certain of them. The first clear conception is due to Goltz, who pointed out that, in the labyrinth and its central connections we had, first, a peripheral sense organ in the semicircular canals and the other portions of the otic labyrinth; second, a centripetal path from this end organ to the central mechanism, and finally certain centrifugal paths from the central mechanism to the muscles, through which the motor response is brought about. The function of the labyrinth is, therefore, a reflex one.

Goltz, however, supposed that the end organs were stimulated by changes in hydrostatic pressure in the labyrinth as the animal moved its head about in space. Although certain remnants of this hydrostatic theory still survive and are likely to survive for some time to come, it has been largely superseded by the kinetic theory of Mach, Breuer and Brown, particularly with reference to the semicircular canals. As Brown expressed it, on the basis of the hydrostatic theory, and static 
theories in general, the labyrinth is regarded as an organ which gives information of the aspect of the head in space. In the kinetic theory, the labyrinth, and especially the semicircular canals, are regarded as an organ which gives information of the change of aspect of the head in space. The distinction is important, for, in response to rapid changes of aspect of the head in space, prompt and rapid motor reactions are necessary to preserve equilibrium or to break a fall. There is no other sense organ in the body which will give this information quite so efficiently as the labyrinth, and hence, there is always a certain amount of permanent deficiency of motor response after total loss of the labyrinths.

Bretuer's original idea was that because of its inertia, the fluid in the canals lagged behind when the head was moved in any plane, and the sensory endings in the labyrinth were stimulated by fluid currents. Mach objected to the currents, arguing that from the point of view of experimental physics, currents were impossible in such fine tubes as the semicircular canals; the effective stimulus must be, therefore, a change in pressure in particular regions of the labyrinth, since the fluid would lag behind sufficiently to cause a change in pressure. Breuer and Brown accepted Mach's modifications of the original theory, and it is generally held to-day that changes in fluid pressure, not fluid current, are the effective or adequate stimuli for the sensory endings in the labyrinth. So far as we can see, it is not necessary to assume the existence of actual currents for the explanation of any known response to stimulation of the labyrinth.

Brown showed that the six canals might be divided into three pairs, each pair lying in a plane at right angles to the planes of the other two pairs. Lee showed that each canal in a given pair had two functions, a principal and a subordinate function; and that the subordinate function of each canal of a pair reinforces the principal function of the other canal. As an illustration of this point we have Breuer's statement that, in the horizontal canals, change of pressure from canal to ampulla is a stronger stimulus than change of pressure from ampulla to canal.

Time does not permit a consideration of some of the "Streitfragen" of labyrinthine physiology. It may be shown that, under certain conditions, the labyrinth exerts an influence on the state of contraction of certain of the muscles of the limbs. How great this influence is under normal conditions is a matter of conjecture. Again, Stefani's idea that the labyrinth exerted its effect mainly over paths passing through the cerebellum is open to question in part at least. Certainly, typical labyrinthine nystagmus may be induced in a dog after complete ablation of the cerebellum. 
Our results are more in line with Luciani's. We believe that while the labyrinthine reaction is primarily a direct reflex effect through the extracerebellar structures, yet the connection with the cerebellum is such that in higher forms at least, the labyrinthine reactions are influenced by the cerebellum.

Finally, the path which labyrinthine impulses follow in going to the cerebrum, or in what part of the cerebral cortex the end stations lie, will be dealt with in a subsequent paper. That labyrinthine impulses do reach the cerebrum is scarcely to be doubted. 